\title{
Optimal Control and Resources
}

\author{
by \\ Michael R. Caputo \\ Department of Economics \\ University of Central Florida \\ P.O. Box 161400 \\ Orlando, FL 32816-1400 \\ email:mcaputo@bus.ucf.edu \\ fax: (407) 823-3269 \\ phone: (407) 823-1405
}

\begin{abstract}
Necessary and sufficient conditions from optimal control theory that are typically used when studying finite and infinite horizon natural resource economics problems are stated. In the finite horizon case their use is demonstrated by deriving an explicit solution of a model describing the optimal cleanup of a hazardous stock. In the infinite horizon case a qualitative characterization of the solution of a renewable resource extraction problem is achieved by employing a phase diagram. Complementary techniques such as comparative dynamics and dynamic envelope results are also employed in order to demonstrate how to extract the maximum amount of information from natural resource economics problems.
\end{abstract}

Key Words: optimal control theory, necessary conditions, sufficient conditions, transversality conditions, comparative dynamics, dynamic envelope theorem, phase diagram

\section{Table of Contents}

1. Introduction and Overview

2. Necessary and Sufficient Conditions For Finite Horizon Optimal Control Problems

3. Optimal Cleanup Of A Hazardous Stock

4. Necessary and Sufficient Conditions for Autonomous and Discounted Infinite Horizon Optimal Control Problems

5. A Renewable Resource Extracting Model of a Firm

6. Summary and Conclusion 
Glossary:

comparative dynamics The process of comparing two dynamic or intertemporal equilibria that result from a change in a parameter.

concave function A differentiable concave function has the property that at each point for which it is defined, the tangent plane to the function lies on or above the function itself.

envelope theorem A theorem that shows how the maximum value of an agent's objective function changes when a parameter changes.

phase diagram A graphical representation of the general solution to a system of differential equations that indicates the change in the solution over time, and from which qualitative features of the solution are readily deduced.

steady state comparative statics The process of comparing two steady state equilibria that result from a change in a parameter.

Cross References:

Managing Stock Pollutants

Taxation of Nonrenewables

Economics of Pest Control

Spatial and Temporal Management of Natural Resources

Differential Games and Resources

The Hartwick Rule

Cartel versus Fringe Models

The Economics of Groundwater

The Economics of Exploration for and Production of Exhaustible Resources

The Economics of Water and Fisheries

The Economics of Market Power on the Extraction of Nonrenewable Resources 


\section{Author Biography:}

Michael R. Caputo earned a B.S. in Economics with distinction from the University of Delaware in 1983, a M.S. in Economics from the University of Washington in 1985, and a Ph.D. in Economics from the University of Washington in 1987. He then obtained a job as an assistant professor in the Department of Agricultural and Resource Economics at the University of California Davis, where he earned tenure and remained for 16 years. Michael married in 1994, and his wife Nora gave birth to a wonderful daughter named Jennifer in 1997. In 2003 Professor Caputo took a faculty position at University of Central Florida. Michael does not like tropical storms and is disappointed by how difficult it is to grow tasty tomatoes in Florida. His hobbies include playing basketball, modifying cars, cooking, dining out, gardening, and "bumping" with Jennifer.

Professor Caputo teaches courses in microeconomic theory and mathematical economics. His research has appeared in numerous peer-reviewed journals, including Review of Economic Studies, Journal of Economic Theory, International Economic Review, Games and Economic Behavior, Journal of Economic Dynamics and Control, Journal of Mathematical Economics, Economic Theory, Journal of Optimization Theory and Applications, Journal of Economics, Metroeconomica, and Journal of Environmental Economics and Management. 


\section{INTRODUCTION AND OVERVIEW}

As a rule, natural resource problems studied by economists are inherently intertemporal or dynamic. As used by resource economists, the word "dynamic" has a specific technical property. A dynamic or intertemporal optimization problem is one in which decisions made in one time period influence those made in a future time period, either directly or indirectly. Such an intertemporal link between decisions is easy to see using the classical Hotelling model of a nonrenewable resource extracting firm. In the said model a firm has a finite amount of time to extract a fixed and finite stock of a natural resource. As a result of this structure, the rate of extraction chosen by a firm "today" determines the quantity of the resource stock available "tomorrow," i.e., the current extraction rate fully determines "tomorrow's" resource stock, which, in turn, influences the rate of extraction "tomorrow" by limiting the amount that can be extracted. In short, the current extraction rate affects the extraction rate in future periods.

It is important to note that the introduction of time, per se, does not necessarily render a natural resource economics problem dynamic. To see this, recall the classical optimal stopping problem, i.e., the optimization problem that dictates when to cut down a tree or how long to age a bottle of wine. Time is integral to such problems-it is the decision variable—-but they are missing the aforementioned intertemporal linkage intrinsic to fundamentally dynamic models.

In the preceding discussion of the Hotelling model, two features are present that speak to the inherently dynamic nature of the optimization problem, to wit, there are two fundamentally different types of variables present. One is the flow or rate variable-the control variable in the language of optimal control theory - that is measured and defined over an interval of time. In the Hotelling model, the extraction rate of the nonrenewable resource is the control variable, and its units might be tons of ore extracted per day. This is the variable that is chosen or controlled by the agent who solves the optimal control problem. The other variable is related to the first but is instead a stock variable — known as a state variable in the parlance of optimal control theorywhich is measured and defined at a point in time. In the Hotelling model, the stock of the nonrenewable resource is the state variable, and its units might be in tons of ore on a given day. The 
link between the two types of variables is that the time rate of change of the state variable is functionally related to the control variable by way of an ordinary differential equation, known as the state equation. Thus choice of the time-path of the control variable fully determines the time path of the state variable by way of the state equation and initial condition on the state variable. For this reason, state and control variables come in pairs, but may differ in their numbers in a given optimal control problem.

With this basic material in hand, this section is brought to a close by setting down the notational conventions used in what follows, namely, (a) all vectors are column vectors and are indicated by boldface type, (b) the derivative of a scalar-valued function with respect to a column vector is a row vector, and is denoted by a boldface letter appearing as a subscript on the said function, (c) the Hessian matrix of a scalar-valued function is indicated by two boldface subscripts on a function, in which case its order is (first subscript order) $\times$ (second subscript order), (d) $\mathbf{0}_{N_{1}}$ is the null column vector in $\mathbb{R}^{N_{1}}$, for example, and (e) " $\dagger$ " denotes transposition.

2. NECESSARY AND SUFFICIENT CONDITIONS FOR FINITE HORIZON OPTIMAL CONTROL PROBLEMS

In the present section and that which ensues, the class of optimal control problems under consideration is as follows:

$$
\begin{gathered}
V(\boldsymbol{\beta}) \stackrel{\text { def }}{=} \max _{\mathbf{u}(\cdot), \mathbf{x}(T)}\left\{\int_{0}^{T} f(t, \mathbf{x}(t), \mathbf{u}(t) ; \boldsymbol{\alpha}) e^{-r t} d t+S(T, \mathbf{x}(T) ; \boldsymbol{\alpha}) e^{-r T}\right\} \\
\text { s.t. } \dot{\mathbf{x}}(t)=\mathbf{g}(t, \mathbf{x}(t), \mathbf{u}(t) ; \boldsymbol{\alpha}), \mathbf{x}(0)=\mathbf{x}_{0},
\end{gathered}
$$

where $\alpha \in \mathbb{R}^{A}$ is a vector of time independent parameters such as market prices or biological parameters, $r \in \mathbb{R}_{+}$is the discount rate, $\mathbf{x}_{0} \in \mathbb{R}^{N}$ is the given, i.e., fixed, initial value of the stock or state vector, $T \in \mathbb{R}_{++}$is the given terminal time of the optimal control problem, and $\boldsymbol{\beta} \stackrel{\text { def }}{=}\left(\mathbf{x}_{0}, T, \boldsymbol{\alpha}, r\right) \in \mathbb{R}^{A+N+2}$. The integrand function $f(\cdot)$ is real-valued and is assumed to be $C^{(1)}$. It may be interpreted as the instantaneous net benefit function at time $t$ given that the state vector has the value $\mathbf{x}(t) \in \mathbb{R}^{N}$ and the control vector has the value $\mathbf{u}(t) \in \mathbb{R}^{M}$. In natural resource problems, $f(t, \mathbf{x}(t), \mathbf{u}(t) ; \boldsymbol{\alpha})$ might be the instantaneous profit flow of a firm or the instantaneous 
felicity flow of an omniscient central planner. The salvage function $S(\cdot)$ imparts a value to the terminal stock $\mathbf{x}(T)$, and is assumed to be $C^{(1)}$. The transition function $\mathbf{g}(\cdot)$ is vector-valued and has values in $\mathbb{R}^{N}$. Each component function of $\mathbf{g}(\cdot)$ is assumed to be $C^{(1)}$ and describes how each state variable changes over time. Finally, the function $V(\cdot)$ is the current value optimal value function. It yields the maximum value of the objective functional given the parameter vector $\boldsymbol{\beta}$ of the optimal control problem, and is fully akin to the indirect profit function or the indirect utility function from neoclassical microeconomic theory. Note that no proofs of the ensuing theorems are offered because the focus of this chapter is on the use of optimal control theory to solve optimal control problems in natural resource economics.

The following definition is basic in optimal control theory.

Definition 2.1. If $\mathbf{u}(\cdot) \in C^{(0)}$ for all $t \in[0, T]$, and $\mathbf{x}(\cdot) \in C^{(1)}$ for all $t \in[0, T]$ is the corresponding state function that satisfies $\dot{\mathbf{x}}(t)=\mathbf{g}(t, \mathbf{x}(t), \mathbf{u}(t) ; \boldsymbol{\alpha})$ and $\mathbf{x}(0)=\mathbf{x}_{0}$, then $(\mathbf{x}(t), \mathbf{u}(t))$ is called an admissible pair.

Definition 2.1 can be relaxed quite a bit. For example, it is common to permit admissible control functions to be piecewise continuous in $t$ and admissible state functions to be piecewise smooth in $t$. The above definition, however, is sufficient for the purposes at hand.

Let $(\mathbf{z}(t ; \boldsymbol{\beta}), \mathbf{v}(t ; \boldsymbol{\beta}))$ be an admissible pair that solves optimal control problem (1), that is, $(\mathbf{z}(t ; \boldsymbol{\beta}), \mathbf{v}(t ; \boldsymbol{\beta}))$ is an optimal pair. Note that the optimal pair is an explicit function of the parameter vector $\beta$. The dependence on $\beta$ is both natural and important, as it paves the way for the dynamic envelope theorem and comparatives dynamics. One final definition will prove useful before the necessary and sufficient conditions are given.

Definition 2.2. The current value Hamiltonian for optimal control problem (1) is defined as

$$
H(t, \mathbf{x}, \mathbf{u}, \lambda ; \boldsymbol{\alpha}) \stackrel{\text { def }}{=} f(t, \mathbf{x}, \mathbf{u} ; \boldsymbol{\alpha})+\lambda^{\dagger} \mathbf{g}(t, \mathbf{x}, \mathbf{u} ; \boldsymbol{\alpha})=f(t, \mathbf{x}, \mathbf{u} ; \boldsymbol{\alpha})+\sum_{n=1}^{N} \lambda_{n} g^{n}(t, \mathbf{x}, \mathbf{u} ; \boldsymbol{\alpha}),
$$


where $\lambda_{i}, i=1,2, \ldots, N$, is the current value costate variable corresponding to the state variable $x_{i}, i=1,2, \ldots, N$.

Observe that for every state variable in an optimal control problem, there corresponds exactly one costate variable.

The following necessary conditions are not the most general available, but are the basic set typically used when optimal control theory is employed to solve finite horizon natural resource economics problems. Note that the ensuing theorems are stated using both vector notation and index notation.

Theorem 2.1. Let $(\mathbf{z}(t ; \boldsymbol{\beta}), \mathbf{v}(t ; \boldsymbol{\beta}))$ be an admissible pair for problem (1). Then if $(\mathbf{z}(t ; \boldsymbol{\beta}), \mathbf{v}(t ; \boldsymbol{\beta}))$ yields the absolute maximum of problem $(1)$, it is necessary that there exist a $C^{(1)}$ function $\lambda(\cdot) \stackrel{\text { def }}{=}\left(\lambda_{1}(\cdot), \lambda_{2}(\cdot), \ldots, \lambda_{N}(\cdot)\right)$ such that for all $t \in[0, T]$,

$$
H_{\mathbf{u}}(t, \mathbf{z}(t ; \boldsymbol{\beta}), \mathbf{v}(t ; \boldsymbol{\beta}), \lambda(t ; \boldsymbol{\beta}) ; \boldsymbol{\alpha})=\mathbf{0}_{M}^{\dagger},
$$

or equivalently

$$
H_{u_{m}}(t, \mathbf{z}(t ; \boldsymbol{\beta}), \mathbf{v}(t ; \boldsymbol{\beta}), \lambda(t ; \boldsymbol{\beta}) ; \boldsymbol{\alpha})=0, m=1,2, \ldots, M \text {, }
$$

and

$$
\dot{\mathbf{z}}(t ; \boldsymbol{\beta})=H_{\lambda}(t, \mathbf{z}(t ; \boldsymbol{\beta}), \mathbf{v}(t ; \boldsymbol{\beta}), \lambda(t ; \boldsymbol{\beta}) ; \boldsymbol{\alpha})^{\dagger}, \mathbf{z}(0 ; \boldsymbol{\beta})=\mathbf{x}_{0},
$$

or equivalently

$$
\dot{z}_{i}(t ; \boldsymbol{\beta})=H_{\lambda_{i}}(t, \mathbf{z}(t ; \boldsymbol{\beta}), \mathbf{v}(t ; \boldsymbol{\beta}), \lambda(t ; \boldsymbol{\beta}) ; \boldsymbol{\alpha}), z_{i}(0 ; \boldsymbol{\beta})=x_{i 0}, i=1,2, \ldots, N,
$$

and

$$
\dot{\lambda}(t ; \boldsymbol{\beta})=r \lambda(t ; \boldsymbol{\beta})-H_{\mathbf{x}}(t, \mathbf{z}(t ; \boldsymbol{\beta}), \mathbf{v}(t ; \boldsymbol{\beta}), \lambda(t ; \boldsymbol{\beta}) ; \boldsymbol{\alpha})^{\dagger}, \lambda(T ; \boldsymbol{\beta})=S_{\mathbf{x}}(T, \mathbf{z}(T ; \boldsymbol{\beta}) ; \boldsymbol{\alpha})^{\dagger},
$$

or equivalently

$$
\dot{\lambda}_{i}(t ; \boldsymbol{\beta})=r \lambda_{i}(t ; \boldsymbol{\beta})-H_{x_{i}}(t, \mathbf{z}(t ; \boldsymbol{\beta}), \mathbf{v}(t ; \boldsymbol{\beta}), \lambda(t ; \boldsymbol{\beta}) ; \boldsymbol{\alpha}), \lambda_{i}(T ; \boldsymbol{\beta})=S_{x_{i}}(T, \mathbf{z}(T ; \boldsymbol{\beta}) ; \boldsymbol{\alpha}), i=1,2, \ldots, N .
$$

Furthermore, if $H(\cdot) \in C^{(2)}$ in $\mathbf{u}$, then

$$
\mathbf{h}^{\dagger} H_{\mathrm{uu}}(t, \mathbf{z}(t ; \boldsymbol{\beta}), \mathbf{v}(t ; \boldsymbol{\beta}), \lambda(t ; \boldsymbol{\beta}) ; \boldsymbol{\alpha}) \mathbf{h} \leq 0 \text { for all } \mathbf{h} \in \mathbb{R}^{M},
$$

or equivalently 


$$
\sum_{\ell=1}^{M} \sum_{m=1}^{M} H_{u_{\ell} u_{m}}(t, \mathbf{z}(t ; \boldsymbol{\beta}), \mathbf{v}(t ; \boldsymbol{\beta}), \lambda(t ; \boldsymbol{\beta}) ; \boldsymbol{\alpha}) h_{\ell} h_{m} \leq 0 \text { for all } \mathbf{h} \in \mathbb{R}^{M}
$$

It is worthwhile to pause and stress four aspects of Theorem 2.1. First, observe that Theorem 2.1 gives a set of necessary conditions that a maximizing pair of curves $(\mathbf{z}(t ; \boldsymbol{\beta}), \mathbf{v}(t ; \boldsymbol{\beta}))$ for problem (1) must satisfy. Note, however, that just because a pair of curves satisfies the necessary conditions of Theorems 2.1, one cannot, at this juncture, be sure that the said pair actually solves the optimal control problem under consideration. In attempting to find a solution to an optimal control problem, one therefore searches for solutions of the necessary conditions, for an optimal solution must satisfy them. In other words, if an admissible pair of curves does not satisfy the necessary conditions, then the pair cannot be the solution to the optimal control problem under consideration.

Second, the necessary conditions in Theorem 2.1 also apply to a minimization problem, save for the last two that involve the quadratic form of the Hessian matrix of $H(\cdot)$ with respect to $\mathbf{u}$. For a minimization problem the weak inequality is reversed for the last two.

Third, note that if the control problem does not include a salvage function, then the necessary condition for the terminal value of the current value costate vector takes the form $\lambda(T ; \boldsymbol{\beta})=\mathbf{0}_{N}$. Terminal conditions on the costate vector are often referred to as transversality conditions. Transversality conditions may be either necessary conditions, as is the case in Theorem 2.1, or they may be sufficient conditions, as in the upcoming Theorem 4.2.

Finally, if one were to use a present value Hamiltonian, namely,

$$
\tilde{H}(t, \mathbf{x}, \mathbf{u}, \lambda ; \boldsymbol{\alpha}) \stackrel{\text { def }}{=} f(t, \mathbf{x}, \mathbf{u} ; \boldsymbol{\alpha}) e^{-r t}+\tilde{\lambda}^{\dagger} \mathbf{g}(t, \mathbf{x}, \mathbf{u} ; \boldsymbol{\alpha})=H(t, \mathbf{x}, \mathbf{u}, \lambda ; \boldsymbol{\alpha}) e^{-r t},
$$

where $\tilde{\lambda} \stackrel{\text { def }}{=} \lambda e^{-r t}$ is the present value costate vector, then the necessary conditions for the costate vector take a slightly different form. In particular, the necessary condition for the present value costate vector takes the form $\dot{\tilde{\lambda}}(t ; \boldsymbol{\beta})=-\tilde{H}_{\mathbf{x}}(t, \mathbf{z}(t ; \boldsymbol{\beta}), \mathbf{v}(t ; \boldsymbol{\beta}), \tilde{\lambda}(t ; \boldsymbol{\beta}) ; \boldsymbol{\alpha})^{\dagger}$, while the transversality condition is given by $\tilde{\lambda}(T ; \boldsymbol{\beta})=e^{-r T} S_{\mathbf{x}}(T, \mathbf{z}(T ; \boldsymbol{\beta}) ; \boldsymbol{\alpha})^{\dagger}$. 
Sufficient conditions for an admissible pair to be optimal can now be stated. They are known as the Mangasarian sufficient conditions.

Theorem 2.2. Let $(\mathbf{z}(t ; \boldsymbol{\beta}), \mathbf{v}(t ; \boldsymbol{\beta}))$ be an admissible pair for problem (1). Suppose that $(\mathbf{z}(t ; \boldsymbol{\beta}), \mathbf{v}(t ; \boldsymbol{\beta}))$ satisfy the necessary conditions of Theorem 2.1 for problem (1) with current value costate vector $\lambda(t ; \boldsymbol{\beta})$. If $H(\cdot)$ is a concave function of $(\mathbf{x}, \mathbf{u})$ for all $t \in[0, T]$ over an open convex set containing all the admissible values of $(\mathbf{x}(\cdot), \mathbf{u}(\cdot))$ when the current value costate vector is $\lambda(t ; \boldsymbol{\beta})$, and $S(\cdot)$ is a concave function of $\mathbf{x}$ over an open convex set containing all the admissible values of $\mathbf{x}(\cdot)$, then $\mathbf{v}(t ; \boldsymbol{\beta})$ is an optimal control and $(\mathbf{z}(t ; \boldsymbol{\beta}), \mathbf{v}(t ; \boldsymbol{\beta}))$ yields the global maximum of problem (1). If $H(\cdot)$ and $S(\cdot)$ are strictly concave functions under the same conditions, then $(\mathbf{z}(t ; \boldsymbol{\beta}), \mathbf{v}(t ; \boldsymbol{\beta}))$ yields the unique global maximum of problem (1).

Essentially, Theorem 2.2 asserts that a solution of the necessary conditions given in Theorem 2.1 is a solution of the posed optimal control problem when the current value Hamiltonian is concave in the state and control vectors and the salvage function is concave in the state vector. In closing, note that if one were dealing with a minimization problem, an analogous theorem holds. Not surprisingly, it follows by replacing the words "concave" with "convex" and "maximum" with "minimum" in Theorem 2.2.

\section{OPTIMAL CLEANUP OF A HAZARDOUS STOCK}

The purpose of this section is to (i) develop an optimal control model that provides a formal description of a typical nonrenewable resource economics problem, (ii) derive the explicit solution of the resulting optimal control problem, (iii) conduct a comparative dynamics analysis of the solution, (iv) derive some envelope results, and (v) provide an economic interpretation of the results in (ii)-(iv). It is important to understand that, in general, it is difficult to find explicit solutions of optimal control models. This fact explains the choice of functional forms used below.

Consider a situation in which a spill of a hazardous substance has occurred at time $t=0$ in the known and fixed amount $x(0)=x_{0}>0$. Let $x(t)$ be the stock of the hazardous substance 
remaining in the environment at time $t$ and $u(t)$ be the rate of cleanup at time $t$. Given these definitions, the stock of the hazardous substance remaining in the environment at time $t$ is given by $x(t) \stackrel{\text { def }}{=} x_{0}-\int_{0}^{t} u(s) d s$. Evaluating the preceding equation at $t=0$ gives $x(0)=x_{0}$, and by applying Leibniz's Rule to it one has $\dot{x}(t)=-u(t)$. The latter two equations are, respectively, the initial condition and the state equation for the ensuing control problem.

Assume that a government agency has contracted with a firm for a fixed interval of time, say $[0, T]$, and known as the planning horizon, to clean up the hazardous substance. Also assume that the contract allows the firm to decide how much of the stock to leave behind at the end of the contract period, i.e., $x(T)$ is a choice variable for the firm.

Suppose that the clean up technology is such that the quadratic cost function $\frac{1}{2} c[u(t)]^{2}$ best describes the per-period cost associated with the clean up process, where $c>0$ is a given parameter. Furthermore, the firm must pay a fine based on how much of the hazardous stock remains in the environment at time $T$, say $S(x(T) ; \tau) \stackrel{\text { def }}{=} \tau x(T)$, where $\tau>0$ is the fine per-unit of the hazardous stock. The objective of the firm is to choose a time-path for the rate of cleanup that minimizes the present discounted cost of cleaning up the hazardous stock over the fixed horizon, plus the present discounted value of the fine associated with the hazardous stock remaining in the environment at time $T$.

Formally, the optimal control problem described above takes the form

$$
\begin{gathered}
C(\boldsymbol{\beta}) \stackrel{\text { def }}{=} \min _{u(\cdot), x(T)}\left\{\int_{0}^{T} \frac{1}{2} c[u(t)]^{2} e^{-r t} d t+\tau x(T) e^{-r T}\right\} \\
\text { s.t. } \dot{x}(t)=-u(t), x(0)=x_{0},
\end{gathered}
$$

where $r>0$ is the discount rate employed by the firm and $\beta \stackrel{\text { def }}{=}\left(x_{0}, T, c, r, \tau\right) \in \mathbb{R}_{++}^{5}$ is the parameter vector of the problem. The nonnegativity constraints on the state and control variables are ignored in what follows because they turn out to never bind under the assumptions adopted. Finally, assume that $x_{0}>c^{-1} r^{-1} \tau e^{-r T}\left[e^{r T}-1\right]$ holds. Its meaning will be revealed in the course of solving problem (3). 
The process of finding the solution of problem (3) begins with the statement of the current value Hamiltonian from Definition 2.2, i.e.,

$$
H(u, \lambda ; c) \stackrel{\text { def }}{=} \frac{1}{2} c u^{2}-\lambda u
$$

where $\lambda$ is the current value costate variable. The economic interpretation of $\lambda$ will be revealed in the course of deriving the solution. Note that the current value Hamiltonian is independent of the stock. This is a result of the assumed functional form for the instantaneous cost function. Also observe that the assumed continuity conditions on the underlying functions hold in this problem.

By Theorem 2.1, the necessary conditions for problem (3) take the form

$$
\begin{gathered}
H_{u}(u, \lambda ; c)=c u-\lambda=0, \\
\dot{\lambda}=r \lambda-H_{x}(u, \lambda ; c)=r \lambda, \lambda(T)=S_{x}(x(T) ; \tau)=\tau, \\
\dot{x}=H_{\lambda}(u, \lambda ; c)=-u, x(0)=x_{0}, \\
H_{u u}(u, \lambda ; c)=c \geq 0 .
\end{gathered}
$$

Before solving the necessary conditions, some qualitative information will be extracted from them. First note that because $c>0$, Eq. (8) holds with a strict inequality. Next, given that $\tau>0, \lambda(T)>0$ follows from the transversality condition in Eq. (6). Furthermore, observe that the costate differential equation in Eq. (6) requires that $\lambda$ and $\dot{\lambda}$ have the same sign, as $r>0$. Given that $\lambda(T)>0$, it then follows that $\lambda(t)>0$ and $\dot{\lambda}(t)>0$ for all $t \in[0, T]$ in an optimal plan. Finally, as $\lambda(t)>0$ for all $t \in[0, T]$ and $c>0$, Eq. (5) implies that $u(t)>0$ for all $t \in[0, T]$ in an optimal plan. In sum, it has been shown that in an optimal clean up plan, the current value costate variable is positive and increasing over the planning horizon, and that the clean up rate is positive in every time period.

In order to solve the necessary conditions, observe that by Eq. (5), the solution for $\lambda$ must first be found in order to determine the solution for $u$. The general solution of Eq. (6) may be found by the method of separation of variables, and is given by $\lambda(t)=a_{1} e^{r t}$, where $a_{1}$ is a constant of integration. The constant $a_{1}$ is found using the transversality condition $\lambda(T)=\tau$, 
which yields $\lambda(T)=a_{1} e^{r T}=\tau$ or $a_{1}=\tau e^{-r T}$. Substituting $a_{1}=\tau e^{-r T}$ in $\lambda(t)=a_{1} e^{r t}$, the specific solution to the costate differential equation is

$$
\lambda(t ; \boldsymbol{\beta})=\tau e^{r[t-T]} .
$$

Using Eq. (9), the solution for the clean up rate can be found from Eq. (5), namely,

$$
v(t ; \boldsymbol{\beta})=c^{-1} \tau e^{r[t-T]} .
$$

Note that the above qualitative conclusions may be verified from these two solutions.

Using Eq. (10), the state equation may be written as $\dot{x}=-c^{-1} \tau e^{r[t-T]}$. Straightforward integration yields its general solution, videlicet $x(t)=-c^{-1} r^{-1} \tau e^{r[t-T]}+a_{2}$, where $a_{2}$ is another constant of integration. The value of $a_{2}$ may be found by employing the general solution and the initial condition $x(0)=x_{0}$, implying that $a_{2}=x_{0}+c^{-1} r^{-1} \tau e^{-r T}$. The specific solution of the state equation is therefore given by

$$
z(t ; \boldsymbol{\beta})=c^{-1} r^{-1} \tau e^{-r T}\left[1-e^{r t}\right]+x_{0} .
$$

In sum, Eqs. (9)-(12) give the solution to the necessary conditions. The amount of the hazardous stock at the end of the planning horizon is therefore given by

$$
z(T ; \boldsymbol{\beta})=c^{-1} r^{-1} \tau e^{-r T}\left[1-e^{r T}\right]+x_{0} .
$$

Note that the inequality $x_{0}>c^{-1} r^{-1} \tau e^{-r T}\left[e^{r T}-1\right]$ is equivalent to $z(T ; \boldsymbol{\beta})>0$.

In order to determine if the above solution of the necessary conditions is a solution of problem (3), the conditions of Theorem 2.2 must be checked. This is a simple matter given the specification of the model, for the salvage function is linear in the hazardous stock and hence convex in it for all $t \in[0, T]$, and as the reader may verify, the current value Hamiltonian is convex in the hazardous stock and clean up rate for all $t \in[0, T]$, independently of the costate variable. Hence by Theorem 2.2, the above solution of the necessary conditions is indeed a solution of problem (3). Furthermore, seeing as there is only one solution of the necessary conditions, it is also the unique solution of problem (3).

At this juncture it is instructive to pause and contemplate how the solution to problem (3) would change if the inequality $x_{0} \geq c^{-1} r^{-1} \tau e^{-r T}\left[e^{r T}-1\right]$ did not hold. In this case, Eq. (12) shows that the hazardous stock would be negative at the terminal time, and therefore by continuity, 
negative for a finite interval of time preceding the terminal time, which does not make sense. In order to solve problem (3) when $x_{0} \geq c^{-1} r^{-1} \tau e^{-r T}\left[e^{r T}-1\right]$ does not hold, one could impose the terminal constraint $x(T) \geq 0$ on the hazardous stock and the nonnegativity constraint $u(t) \geq 0$ on the clean up rate. Given the state equation $\dot{x}(t)=-u(t)$ and constraint $u(t) \geq 0$, it follows that if $x(T) \geq 0$, then $x(t) \geq 0$ for all $t \in[0, T]$, i.e., imposing that the clean up rate is nonnegative throughout the planning horizon and that the terminal value of the hazardous stock is nonnegative, ensures that the hazardous stock is nonnegative throughout the planning horizon.

The qualitative nature of the solution under these modifications may be ascertained without the use of necessary and sufficient conditions that formally account for the terminal constraint $x(T) \geq 0$ and the nonnegativity constraint $u(t) \geq 0$. To begin, observe that if the constraint $x(T) \geq 0$ binds - the case of interest under the present stipulations-there must be an interval of time in which $u(t)=0$, otherwise the hazardous waste stock would turn negative, violating $x(T) \geq 0$. Now recall that the objective of the firm is to minimize the presented discounted value of its clean up costs. This implies that the firm favors pushing costs to the future. In turn, this means that the firm would choose to set $u(t)=0$ during an initial interval of the planning horizon, say $\left[0, t^{*}\right]$, where $t^{*} \in(0, T)$. By doing so, a lower present value of cost is achieved than if the firm were to choose $u(t)=0$ in some interval of time near the terminal time and thereby incur clean up costs early in the planning period. In sum, the optimal plan would involve cleanup during the interval $\left(t^{*}, T\right]$, with the hazardous stock declining monotonically to zero at $T$, whereas no cleanup would take place in the initial interval $\left[0, t^{*}\right]$, thereby implying that the hazardous stock would remain at its initial value during this period. With this matter dispensed with, attention is turned back to problem (3) under the original assumptions.

Before turning to the economic interpretation of the solution and the comparative dynamics, the minimum present value cost of the clean up plan will be calculated. By definition, the said cost is given by substituting the optimal pair $(z(t ; \boldsymbol{\beta}), v(t ; \boldsymbol{\beta}))$ in the objective functional and performing the indicated operations, that is, 


$$
\begin{aligned}
C(\boldsymbol{\beta}) & \stackrel{\text { def }}{=} \int_{0}^{T} \frac{1}{2} c[v(t ; \boldsymbol{\beta})]^{2} e^{-r t} d t+\tau z(T ; \boldsymbol{\beta}) e^{-r T} \\
& =\int_{0}^{T} \frac{1}{2} c\left[c^{-1} \tau e^{r[t-T]}\right]^{2} e^{-r t} d t+\tau\left[c^{-1} r^{-1} \tau e^{-r T}\left[1-e^{r T}\right]+x_{0}\right] e^{-r T} \\
& =\frac{1}{2} c^{-1} \tau^{2} e^{-2 r T} \int_{0}^{T} e^{r t} d t+c^{-1} r^{-1} \tau^{2} e^{-2 r T}\left[1-e^{r T}\right]+\tau x_{0} e^{-r T} \\
& =\frac{1}{2} c^{-1} r^{-1} \tau^{2} e^{-2 r T}\left[e^{r T}-1\right]+c^{-1} r^{-1} \tau^{2} e^{-2 r T}\left[1-e^{r T}\right]+\tau x_{0} e^{-r T} \\
& =\frac{1}{2} c^{-1} r^{-1} \tau^{2} e^{-2 r T}\left[1-e^{r T}\right]+\tau x_{0} e^{-r T} .
\end{aligned}
$$

Recalling that $x_{0}>c^{-1} r^{-1} \tau e^{-r T}\left[e^{r T}-1\right]$, it follows that $C(\boldsymbol{\beta})>0$.

Examination of the optimal clean up rate in Eq. (10) shows that it increases over time, i.e., $\dot{v}(t ; \boldsymbol{\beta})=c^{-1} r \tau e^{r[t-T]}>0$. This is not surprising, in that with a positive rate of discount it behooves the firm to push its costs, and hence the bulk of its cleanup, towards the future relative to the present. It also follows from Eq. (11) that the hazardous stock decreases monotonically over the planning horizon, that is, $\dot{z}(t ; \boldsymbol{\beta})=-c^{-1} \tau e^{-r T} e^{r t}<0$. Moreover, recalling that $z(T ; \boldsymbol{\beta})>0$, the least cost clean up plan has the firm paying a fine rather than fully cleaning up the hazardous stock under the given stipulations.

Regarding the solution for the current value costate variable, first note that

$$
C_{x_{0}}(\boldsymbol{\beta})=\frac{\partial}{\partial x_{0}}\left[\frac{1}{2} c^{-1} r^{-1} \tau^{2} e^{-2 r T}\left[1-e^{r T}\right]+\tau x_{0} e^{-r T}\right]=\tau e^{-r T}=\lambda(0 ; \boldsymbol{\beta})>0
$$

follows from Eqs. (9) and (13). Equation (14) shows that the current value costate variable in the initial period of the planning horizon is equal to the marginal cost of the initial hazardous stock. This result is a special case of the dynamic envelope theorem. More generally, the current value costate variable at time $t$ can be interpreted as the current value shadow price of the corresponding state variable at time $t$. In the present model, the current value costate variable has the interpretation of the current value marginal cost of the hazardous stock because it is a cost minimization problem. Using Eq. (9), it follows that the current value marginal cost of the hazardous stock increases over the planning horizon at the firm's rate of discount, i.e., $\dot{\lambda}(t ; \boldsymbol{\beta}) / \lambda(t ; \boldsymbol{\beta})=r$, which is the basic Hotelling rule. It is not surprising that the Hotelling rule holds, seeing as there 
is no stock effect in the instantaneous cost function and problem (3) is just a cost minimization version of the classical Hotelling nonrenewable resource extraction problem.

The comparative dynamics of the hazardous waste clean up problem are easily determined seeing as an explicit solution has been found. This is rare in optimal control problems, and thus attests to the simplicity of the chosen functional forms. For example, Eqs. (9) and (10) show that the current value marginal cost of the stock and the optimal clean up rate are independent of the initial stock. As a result of the latter, the terminal hazardous stock increases by the size of the increase in the initial stock, i.e., $\partial z(T ; \boldsymbol{\beta}) / \partial x_{0}=1$ by Eq. (12). Furthermore, by Eq. (14), the firm's minimum cost of cleanup increases as a result. That the optimal clean up policy is unaffected by the size of the initial spill is a result of the fact that the instantaneous cost function is independent of the hazardous stock, and does not necessarily hold in a more general version of the model.

A question of interest is: If the firm were offered a longer time to cleanup, would it in fact clean up more? By Eq. (12), $\partial z(T ; \beta) / \partial T=-c^{-1} \tau e^{-r T}<0$, hence the longer time permitted for cleaning up the stock does indeed result in a smaller terminal stock. The firm accomplishes this by cleaning up at a slower rate over a longer period of time, thereby resulting in a lower current value marginal cost of the stock, as may be confirmed by $\partial v(t ; \boldsymbol{\beta}) / \partial T=-c^{-1} r \tau e^{r[t-T]}<0$ and $\partial \lambda(t ; \boldsymbol{\beta}) / \partial T=-r \tau e^{r[t-T]}<0$, respectively. Given the assumption that $x_{0}>c^{-1} r^{-1} \tau e^{-r T}\left[e^{r T}-1\right]$, the minimum cost of the resulting clean up plan is lower as well, in view of the fact that $C_{T}(\boldsymbol{\beta})=-r \tau e^{-r T}\left[x_{0}-c^{-1} r^{-1} \tau e^{-r T}\left[\frac{1}{2} e^{r T}-1\right]\right]<0$. In sum, the longer contact is better for the firm and the environment.

The final comparative dynamics results to be discussed are those that pertain to a change in the fine. Economic intuition suggests that a higher fine will lead to a higher clean up rate and thus greater cumulative cleanup, a higher current value marginal cost of the stock, and an increase in the minimum cost of the clean up plan. This intuition is confirmed by the following results: $\partial v(t ; \boldsymbol{\beta}) / \partial \tau=c^{-1} e^{r[t-T]}>0, \quad \partial z(T ; \boldsymbol{\beta}) / \partial \tau=c^{-1} r^{-1} e^{-r T}\left[1-e^{r T}\right]<0, \quad \partial \lambda(t ; \boldsymbol{\beta}) / \partial \tau=e^{r[t-T]}>0$, 
and $C_{\tau}(\boldsymbol{\beta})=e^{-r T}\left[x_{0}-c^{-1} r^{-1} \tau e^{-r T}\left[e^{r T}-1\right]\right]>0$, the latter following from the assumption that $x_{0}>c^{-1} r^{-1} \tau e^{-r T}\left[e^{r T}-1\right]$.

In passing, it is worthwhile to reconsider the hazardous waste problem under the more general assumption that the per-period cost function is of the form $\frac{1}{2} c[u(t)]^{2}+\theta x(t)$, where $\theta>0$. The inclusion of the waste stock and the parameter $\theta$ in the instantaneous cost function might reflect a fine that the firm pays in each period due to the environmental damages created by the hazardous stock.

\section{NECESSARY AND SUFFICIENT CONDITIONS FOR AUTONOMOUS AND DISCOUNTED INFINITE HORIZON OPTIMAL CONTROL PROBLEMS}

The class of optimal control problems under consideration in this section and the next is ubiquitous in economics, namely, the discounted and autonomous family of infinite horizon optimal control problems defined by

$$
\begin{aligned}
& V(\boldsymbol{\beta}) \stackrel{\text { def }}{=} \max _{\mathbf{u}(\cdot)} \int_{0}^{+\infty} f(\mathbf{x}(t), \mathbf{u}(t) ; \boldsymbol{\alpha}) e^{-r t} d t \\
& \text { s.t. } \quad \dot{\mathbf{x}}(t)=\mathbf{g}(\mathbf{x}(t), \mathbf{u}(t) ; \boldsymbol{\alpha}), \mathbf{x}(0)=\mathbf{x}_{0},
\end{aligned}
$$

where now $r \in \mathbb{R}_{++}$and all terms are as defined in $\S 2$, save for $\boldsymbol{\beta} \stackrel{\text { def }}{=}\left(\mathbf{x}_{0}, \boldsymbol{\alpha}, r\right) \in \mathbb{R}^{N+A+1}$, and where the reader should note the slight abuse of notation. Problem (15) is an autonomous control problem because the integrand and transition functions do not depend explicitly on the independent variable $t$. This is one feature of problem (15) that distinguishes it from problem (1). Another is that problem (15) has an infinite planning horizon while problem (1) has a finite planning horizon. Because of this difference, the salvage function from problem (1) no longer exists in problem (15). Finally, the definition of an admissible pair for problem (15) is taken to coincide with Definition 2.1, with the exception that $T$ is replaced with $+\infty$. As before, this definition can be considerably relaxed, but is sufficient for the purposes at hand.

Two technical matters arise because of the assumption of an infinite planning horizon. The first is whether or not the objective functional exists, i.e., converges, as it is now an indefinite integral and does not necessarily exist for all admissible pairs under the stipulation placed on 
$f(\cdot)$ in $\S 2$. This technical (but important) matter is sidestepped here by assuming that the objective functional converges for all admissible pairs. Indeed, it is advisable, at least initially, to proceed by solving infinite horizon optimal control problems under this assumption. If convergence fails to occur, then one would look for weaker optimality criteria for which an optimum may exist. It turns out, however, that the exponential discounting, which is ubiquitous in resource economics models, makes this assumption less extreme than it may appear. To see this, first observe that $e^{-r t} \rightarrow 0$ as $t \rightarrow+\infty$. Thus, as long as $f(\cdot)$ is bounded, the objective functional in problem (15) will converge for all admissible pairs. The second technical matter pertains to the necessary conditions for problem (15), and will be discussed after they are given.

Given the autonomous nature of problem (15), it is worthwhile to remark that the current value Hamiltonian is now defined as

$$
H(t, \mathbf{x}, \mathbf{u}, \lambda ; \boldsymbol{\alpha}) \stackrel{\text { def }}{=} f(\mathbf{x}, \mathbf{u} ; \boldsymbol{\alpha})+\lambda^{\dagger} \mathbf{g}(\mathbf{x}, \mathbf{u} ; \boldsymbol{\alpha})=f(\mathbf{x}, \mathbf{u} ; \boldsymbol{\alpha})+\sum_{n=1}^{N} \lambda_{n} g^{n}(\mathbf{x}, \mathbf{u} ; \boldsymbol{\alpha}) .
$$

The necessary conditions for problem (15) are summarized in the following theorem.

Theorem 4.1. Let $(\mathbf{z}(t ; \boldsymbol{\beta}), \mathbf{v}(t ; \boldsymbol{\beta}))$ be an admissible pair for problem (15). Then if $(\mathbf{z}(t ; \boldsymbol{\beta}), \mathbf{v}(t ; \boldsymbol{\beta}))$ yields the absolute maximum of problem (15), it is necessary that there exist a $C^{(1)}$ function $\lambda(\cdot) \stackrel{\text { def }}{=}\left(\lambda_{1}(\cdot), \lambda_{2}(\cdot), \ldots, \lambda_{N}(\cdot)\right)$ such that for all $t \in[0,+\infty)$,

$$
H_{\mathbf{u}}(\mathbf{z}(t ; \boldsymbol{\beta}), \mathbf{v}(t ; \boldsymbol{\beta}), \lambda(t ; \boldsymbol{\beta}) ; \boldsymbol{\alpha})=\mathbf{0}_{M}^{\dagger},
$$

or equivalently

$$
H_{u_{m}}(\mathbf{z}(t ; \boldsymbol{\beta}), \mathbf{v}(t ; \boldsymbol{\beta}), \lambda(t ; \boldsymbol{\beta}) ; \boldsymbol{\alpha})=0, m=1,2, \ldots, M,
$$

and

$$
\dot{\mathbf{z}}(t ; \boldsymbol{\beta})=H_{\lambda}(\mathbf{z}(t ; \boldsymbol{\beta}), \mathbf{v}(t ; \boldsymbol{\beta}), \lambda(t ; \boldsymbol{\beta}) ; \boldsymbol{\alpha})^{\dagger}, \mathbf{z}(0 ; \boldsymbol{\beta})=\mathbf{x}_{0}
$$

or equivalently

$$
\dot{z}_{i}(t ; \boldsymbol{\beta})=H_{\lambda_{i}}(\mathbf{z}(t ; \boldsymbol{\beta}), \mathbf{v}(t ; \boldsymbol{\beta}), \lambda(t ; \boldsymbol{\beta}) ; \boldsymbol{\alpha}), z_{i}(0 ; \boldsymbol{\beta})=x_{i 0}, i=1,2, \ldots, N,
$$

and

$$
\dot{\lambda}(t ; \boldsymbol{\beta})=r \lambda(t ; \boldsymbol{\beta})-H_{\mathbf{x}}(\mathbf{z}(t ; \boldsymbol{\beta}), \mathbf{v}(t ; \boldsymbol{\beta}), \lambda(t ; \boldsymbol{\beta}) ; \boldsymbol{\alpha})^{\dagger}
$$

or equivalently 


$$
\dot{\lambda}_{i}(t ; \boldsymbol{\beta})=r \lambda_{i}(t ; \boldsymbol{\beta})-H_{x_{i}}(\mathbf{z}(t ; \boldsymbol{\beta}), \mathbf{v}(t ; \boldsymbol{\beta}), \lambda(t ; \boldsymbol{\beta}) ; \boldsymbol{\alpha}), i=1,2, \ldots, N
$$

and

$$
\lim _{t \rightarrow+\infty} H(\mathbf{z}(t ; \boldsymbol{\beta}), \mathbf{v}(t ; \boldsymbol{\beta}), \lambda(t ; \boldsymbol{\beta}) ; \boldsymbol{\alpha}) e^{-r t}=0 .
$$

Furthermore, if $H(\cdot) \in C^{(2)}$ in $\mathbf{u}$, then

$$
\mathbf{h}^{\dagger} H_{\mathrm{uu}}(\mathbf{z}(t ; \boldsymbol{\beta}), \mathbf{v}(t ; \boldsymbol{\beta}), \lambda(t ; \boldsymbol{\beta}) ; \boldsymbol{\alpha}) \mathbf{h} \leq 0 \text { for all } \mathbf{h} \in \mathbb{R}^{M},
$$

or equivalently

$$
\sum_{\ell=1}^{M} \sum_{m=1}^{M} H_{u_{\ell} u_{m}}(\mathbf{z}(t ; \boldsymbol{\beta}), \mathbf{v}(t ; \boldsymbol{\beta}), \lambda(t ; \boldsymbol{\beta}) ; \boldsymbol{\alpha}) h_{\ell} h_{m} \leq 0 \text { for all } \mathbf{h} \in \mathbb{R}^{M}
$$

There are two features of Theorem 4.1 that differ from Theorem 2.1, its finite horizon counterpart. First, given the infinite planning horizon and the absence of a salvage function in problem (15), and then recalling the last remark made after Theorem 2.1, it is natural to expect that a necessary condition might be $\lim _{t \rightarrow+\infty} \lambda(t ; \boldsymbol{\beta}) e^{-r t}=\lim _{t \rightarrow+\infty} \tilde{\lambda}(t ; \boldsymbol{\beta})=\mathbf{0}_{N}$. This condition, however, is not in general necessary. Indeed, it is important to understand that, in general, there is no necessary condition on the limiting value of the current value costate variable in Theorem 4.1. Instead, the infinite horizon transversality condition applies to the present value Hamiltonian evaluated along the optimal path-its limit is zero. Note that the aforesaid condition is necessary only for the class of optimal control problems defined by problem (15).

A useful set of sufficient conditions can now be stated for problem (15). Note that this theorem contains a transversality condition that applies to the current value costate vector.

Theorem 4.2. Let $(\mathbf{z}(t ; \boldsymbol{\beta}), \mathbf{v}(t ; \boldsymbol{\beta}))$ be an admissible pair for problem (15). Suppose that $(\mathbf{z}(t ; \boldsymbol{\beta}), \mathbf{v}(t ; \boldsymbol{\beta}))$ satisfy the necessary conditions of Theorem 4.1 for problem (15) with current value costate vector $\lambda(t ; \boldsymbol{\beta})$, save for the limiting transversality condition on $H(\cdot)$. If $H(\cdot)$ is a concave function of $(\mathbf{x}, \mathbf{u})$ for all $t \in[0,+\infty)$ over an open convex set containing all the admissible values of $(\mathbf{x}(\cdot), \mathbf{u}(\cdot))$ when the current value costate vector is $\lambda(t ; \boldsymbol{\beta})$, and iffor every admissible control path $\mathbf{u}(t), \lim _{t \rightarrow+\infty} e^{-r t} \lambda(t ; \boldsymbol{\beta})^{\dagger}[\mathbf{z}(t ; \boldsymbol{\beta})-\mathbf{x}(t)] \leq 0$, where $\mathbf{x}(t)$ is the time path of the 
state variable corresponding to $\mathbf{u}(t)$, then $\mathbf{v}(t ; \boldsymbol{\beta})$ is an optimal control and $(\mathbf{z}(t ; \boldsymbol{\beta}), \mathbf{v}(t ; \boldsymbol{\beta}))$ yields the global maximum of problem (15). If $H(\cdot)$ is a strictly concave function under the same conditions, then $(\mathbf{z}(t ; \boldsymbol{\beta}), \mathbf{v}(t ; \boldsymbol{\beta}))$ yields the unique global maximum of problem (15).

The main difference between Theorems 4.2 and 2.2 lies with the transversality condition on the current value costate vector. In Theorem 2.2, sufficiency is obtained when the necessary conditions are satisfied by an admissible pair and the current value Hamiltonian is concave in the state and control variables. In contrast, Theorem 4.2 asserts that satisfaction of the necessary conditions and concavity of the current value Hamiltonian is not enough, because the necessary conditions do not include a transversality condition on the current value costate vector. Thus Theorem 4.2 requires such a condition for sufficiency to obtain. The next section makes use of Theorems 4.1 and 4.2 to qualitatively solve a prototypical renewable resource extraction problem in economics.

\section{A RENEWABLE RESOURCE EXTRACTING MODEL OF A FIRM}

To keep matters relatively simple, and to sharpen the qualitative conclusions that may be drawn, the renewable resource extraction problem analyzed in this section concerns a firm that has the property rights to extract water from an aquifer under conditions of certainty. The presentation of the model can be simplified if some basic duality theory from neoclassical microeconomics is employed. To that end, define the restricted cost function $C(\cdot) \in C^{(2)}$ by

$$
C(x, q ; \mathbf{w}) \stackrel{\text { def }}{=} \min _{\mathbf{v} \in \mathbb{R}_{++}^{N}}\left[\mathbf{w}^{\dagger} \mathbf{v} \text { s.t. } f(x, \mathbf{v}) \geq q\right],
$$

where $x$ is the stock of water in the aquifer, $\mathbf{v}$ is a vector of variable inputs used to extract the water from the aquifer using the extraction production function $f(\cdot) \in C^{(2)}, q$ is the rate of extraction of the water, or the rate of output from the extraction production function, and $\mathbf{w} \in \mathbb{R}_{++}^{N}$ is the vector of market-determined prices of the variable inputs. The value $C(x, q ; \mathbf{w})$ is, by definition, the minimum total cost of extracting water at the rate $q$, given the stock of water $x$ and market prices of the variable inputs $\mathbf{w} \in \mathbb{R}_{++}^{N}$. It is typical to assume that the marginal cost 
of extraction is positive, i.e., $C_{q}(x, q ; \mathbf{w})>0$, and that total and marginal extraction costs increase as the stock of water is drawn down, that is, $C_{x}(x, q ; \mathbf{w})<0$ and $C_{q x}(x, q ; \mathbf{w})<0$, respectively. The latter two inequalities are the archetype "stock effect" assumptions invoked in the resource economics literature. The price vector $\mathbf{w}$ is not germane in what follows and will henceforth be suppressed from the cost function.

It is assumed that the aquifer recharges at the known rate $R>0$, and is offset by the rate at which water is withdrawn, namely $q(t)$. Hence, on net, the time rate of change of the stock of water in the aquifer at time $t$ is given by $\dot{x}(t)=R-q(t)$, and $x(0)=x_{0}$ is the initial stock of water. The firm in question is assumed to take the constant price of the extracted water as given at $p>0$. Thus the firm is assumed to be a price taker and have static expectations with respect to the output price. Finally, the discount rate used by the firm is $r>0$.

Putting the above information together, the optimal control problem facing the firm is

$$
\begin{gathered}
\Pi(\boldsymbol{\beta}) \stackrel{\text { def }}{=} \max _{q(\cdot)} \int_{0}^{+\infty}[p q(t)-C(x(t), q(t))] e^{-r t} d t \\
\dot{x}(t)=R-q(t), x(0)=x_{0},
\end{gathered}
$$

where $\boldsymbol{\beta} \stackrel{\text { def }}{=}\left(x_{0}, p, r, R\right) \in \mathbb{R}_{++}^{4}$. The optimal value function $\Pi(\cdot)$ gives the maximum present discounted value of profit that the firm can earn, given the initial size of the water stock, the market price of the extracted water, the discount rate, and the natural recharge rate of the aquifer. The natural nonnegativity constraints $x(t) \geq 0$ and $q(t) \geq 0$ are assumed not to bind along the optimal path.

Defining the current value Hamiltonian as

$$
H(x, q, \lambda ; p, R) \stackrel{\text { def }}{=} p q-C(x, q)+\lambda[R-q],
$$

it follows from Theorem 4.1 that the necessary conditions of problem (17) are given by

$$
\begin{gathered}
H_{q}(x, q, \lambda ; p, R)=p-C_{q}(x, q)-\lambda=0, \\
\dot{\lambda}=r \lambda-H_{x}(x, q, \lambda ; p, R)=r \lambda+C_{x}(x, q), \\
\dot{x}=H_{\lambda}(x, q, \lambda ; p, R)=R-q, x(0)=x_{0}, \\
\lim _{t \rightarrow+\infty} e^{-r t} H(x, q, \lambda ; p, R)=\lim _{t \rightarrow+\infty} e^{-r t}[p q-C(x, q)+\lambda[R-q]]=0, \\
H_{q q}(x, q, \lambda ; p, R)=-C_{q q}(x, q) \leq 0 .
\end{gathered}
$$


In the present model, the current value costate variable $\lambda(t)$ has the economic interpretation of the current value shadow price of the stock of water at time $t$, i.e., it is the increase in the firm's maximum present discounted value of profit as a result of a marginal increase in the stock of water in the aquifer at time $t$. Naturally, one expects it to be positive, seeing as the stock of water is the source of the firm's profit, intuition that will be confirmed in due course.

Turning to the interpretation of the necessary conditions, Eq. (19) asserts that the optimal rate of extraction equates the market price of the extracted water with the marginal cost of extracting the water, plus the current value shadow price of water. This implies that for a given stock of water, the optimal intertemporal extraction rate is less than the rate that a myopic firm would choose, as the former accounts for the opportunity cost of extracting the water while the latter does not. Equation (20) is best interpreted after Theorem 4.2 is examined. Equation (22) asserts that in an optimal extraction plan, the present value of the firm's instantaneous profit flow plus the imputed value of the net flow of water vanish in the limit of the planning horizon. Finally, Eq. (23) asserts that the cost function is locally concave in the extraction rate in an optimal plan.

Given that the functional form of the cost function is unspecified, an explicit solution of problem (17) is not possible. As a result, it is natural to inquire if a solution of the necessary conditions, assuming one exists, is a solution problem (17). Theorem 4.2 provides a set of conditions under which this is indeed the case. To examine this matter, assume that an admissible solution of the necessary conditions exists, say $\left(x^{*}(t ; \boldsymbol{\beta}), q^{*}(t ; \boldsymbol{\beta})\right)$, with corresponding current value shadow price $\lambda(t ; \boldsymbol{\beta})$, such that it converges to a finite steady state solution of the necessary conditions as $t \rightarrow+\infty$. Denote the steady state solution of the necessary conditions by the triplet $\left(x^{s}(\boldsymbol{\theta}), q^{s}(\boldsymbol{\theta}), \lambda^{s}(\boldsymbol{\theta})\right)$, where $\boldsymbol{\theta} \stackrel{\text { def }}{=}(p, r, R) \in \mathbb{R}_{++}^{3}$, and note that the triplet is a solution of Eqs. (19) -(21) when $\dot{\lambda}=0$ and $\dot{x}=0$. As $\dot{\lambda}=0$ and $\dot{x}=0$ hold in a steady state, $\left(x^{s}(\boldsymbol{\theta}), q^{s}(\boldsymbol{\theta}), \lambda^{s}(\boldsymbol{\theta})\right)$ is a constant solution with respect to time but will in general change as the parameters change, as the notation indicates. In sum, it is assumed that $\left(x^{*}(t ; \boldsymbol{\beta}), q^{*}(t ; \boldsymbol{\beta}), \lambda(t ; \boldsymbol{\beta})\right) \rightarrow\left(x^{s}(\boldsymbol{\theta}), q^{s}(\boldsymbol{\theta}), \lambda^{s}(\boldsymbol{\theta})\right)$ as $t \rightarrow+\infty$. Note that such a solution of necessary conditions (19)-(21) also satisfies the neces- 
sary condition (22), because as $t \rightarrow+\infty,\left(x^{*}(t ; \boldsymbol{\beta}), q^{*}(t ; \boldsymbol{\beta}), \lambda(t ; \boldsymbol{\beta})\right) \rightarrow\left(x^{s}(\boldsymbol{\theta}), q^{s}(\boldsymbol{\theta}), \lambda^{s}(\boldsymbol{\theta})\right)$ and $e^{-r t} \rightarrow 0$

Theorem 4.2 asserts that the above solution of the necessary conditions is indeed a solution of the posed control problem if (i) the current value Hamiltonian is a concave function of the stock of water and the extraction rate, and (ii) $\lim _{t \rightarrow+\infty} e^{-r t} \lambda(t ; \boldsymbol{\beta})\left[x^{*}(t ; \boldsymbol{\beta})-x(t)\right] \leq 0$ for all admissible time-paths of the stock of water $x(t)$. Regarding the former condition, it is a simple exercise to confirm that it holds if and only if the cost function is convex in the water stock and extraction rate. As to the latter, it will hold if all admissible time-paths of the stock of water are either bounded or $\lim _{t \rightarrow+\infty} x(t)$ exists for all admissible time-paths, in view of the fact that $\left(x^{*}(t ; \boldsymbol{\beta}), \lambda(t ; \boldsymbol{\beta})\right) \rightarrow\left(x^{s}(\boldsymbol{\theta}), \lambda^{s}(\boldsymbol{\theta})\right)$ and $e^{-r t} \rightarrow 0$ as $t \rightarrow+\infty$. These two assumptions will be maintained in what follows, thus permitting the claim that $\left(x^{*}(t ; \boldsymbol{\beta}), q^{*}(t ; \boldsymbol{\beta})\right)$ is the optimal pair for problem (17), with corresponding current value shadow price of the water stock $\lambda(t ; \boldsymbol{\beta})$. It is also assumed that $C(\cdot)$ is strongly convex in $(x, q)$, i.e., $C_{x x}(x, q)>0, C_{q q}(x, q)>0$, and $C_{x x}(x, q) C_{q q}(x, q)-\left[C_{x q}(x, q)\right]^{2}>0$, thus implying that the said solution is unique in view of the fact that a strongly convex function is strictly convex.

The above claim that the current value shadow price of the water stock is positive in an optimal plan will now be verified. It is easy to show that the steady state value of the said shadow price is positive, i.e., $\lambda^{s}(\boldsymbol{\theta})>0$. Recalling that $\dot{\lambda}=0$ in a steady state and that $C_{x}(x, q)<0$, it follows from Eq. (20) that $\lambda=-r^{-1} C_{x}(x, q)>0$ in a steady state. In order to show that $\lambda(t ; \beta)>0$ for all $t \in[0,+\infty)$, integrate Eq. (20) forward in time from $t$ to $+\infty$, and then use the fact that $\lambda(t ; \boldsymbol{\beta}) \rightarrow \lambda^{s}(\boldsymbol{\theta})$ as $t \rightarrow+\infty$ to set the constant of integration to zero to get $\lambda(t)=-\int_{t}^{+\infty} e^{-r[s-t]} C_{x}(x(s), q(s)) d s>0$. The last formula for the current value shadow price of the water stock also provides an alternative way to interpret it.

With the above matters addressed, attention is turned to extracting qualitative information about the unique solution using a phase diagram. In order to draw the phase diagram, the three necessary conditions in Eqs. (19)-(21) must be reduced to a pair of ordinary differential equations. There are two pairs of variables that can be used in constructing the phase diagram, to wit, 
$(x, q)$ or $(x, \lambda)$. Formally, it does not matter which pair is chosen for the construction of the phase diagram. This follows from the fact that the information contained in either pair of differential equations is identical, as both sets of differential equations are derived from the same three necessary conditions. The choice of which pair to employ should be made based on the questions of interest. For the present exercise, the $(x, q)$ pair is used.

Because the state equation involves only the two variables of interest, it is already in the correct form for the construction of a phase diagram in the $(x, q)$ phase space. The task at hand, therefore, is the derivation of an ordinary differential equation for $q$ that involves only the pair of variables $(x, q)$. To begin the derivation, note that necessary condition (19) holds as an identity in $t$ when evaluated along the optimal solution paths. As a result, it may be differentiated with respect to $t$ to get

$$
\frac{d}{d t}\left[p-C_{q}(x, q)-\lambda\right]=-C_{q x}(x, q) \dot{x}-C_{q q}(x, q) \dot{q}-\dot{\lambda}=0 .
$$

Equation (19) may also be solved for $\lambda$ to arrive at

$$
\lambda=p-C_{q}(x, q) \text {. }
$$

Upon substituting the state equation (21) and the costate equation (20) in Eq. (24), replacing $\lambda$ in the resulting equation with Eq. (25), and then solving for $\dot{q}$, the pair of differential equations of interest are found to be

$$
\begin{gathered}
\dot{x}=R-q, \\
\dot{q}=\frac{C_{q x}(x, q)[R-q]+r\left[p-C_{q}(x, q)\right]+C_{x}(x, q)}{-C_{q q}(x, q)} .
\end{gathered}
$$

Equations (26) and (27) form the basis for the phase diagram that follows. It is important to recognize that the steady state solution $\left(x^{s}(\boldsymbol{\theta}), q^{s}(\boldsymbol{\theta})\right)$ is the solution to Eqs. (26) and (27) when $\dot{x}=0$ and $\dot{q}=0$.

The construction of the phase diagram in the $(x, q)$ phase space begins with the calculation of the Jacobian matrix of the dynamical system given in Eqs. (26) and (27), i.e., 


$$
J_{d} \stackrel{\text { def }}{=}\left[\left.\begin{array}{ll}
\frac{\partial \dot{x}}{\partial x} & \frac{\partial \dot{x}}{\partial q} \\
\frac{\partial \dot{q}}{\partial x} & \frac{\partial \dot{q}}{\partial q}
\end{array}\right|_{\substack{\dot{x}=0 \\
\dot{q}=0}}=\left[\begin{array}{cc}
0 & -1 \\
\frac{-r C_{q x}\left(x^{s}(\boldsymbol{\theta}), q^{s}(\boldsymbol{\theta})\right)+C_{x x}\left(x^{s}(\boldsymbol{\theta}), q^{s}(\boldsymbol{\theta})\right)}{-C_{q q}\left(x^{s}(\boldsymbol{\theta}), q^{s}(\boldsymbol{\theta})\right)} & r
\end{array}\right] .\right.
$$

Recalling that $C_{x x}(x, q)>0, C_{q q}(x, q)>0$, and $C_{q x}(x, q)<0$, it follows that $\left|J_{d}\right|<0$. By a wellknown theorem from the theory of ordinary differential equations, $\left|J_{d}\right|<0$ implies that the steady state solution $\left(x^{s}(\boldsymbol{\theta}), q^{s}(\boldsymbol{\theta})\right)$ is a local saddle point, with all but one pair of trajectories in the phase plane moving away from the steady state as $t \rightarrow+\infty$. The pair of trajectories that converge to $\left(x^{s}(\boldsymbol{\theta}), q^{s}(\boldsymbol{\theta})\right)$ as $t \rightarrow+\infty$ represent the unique optimal solution to problem (17). The goal in what follows is to identify these trajectories in the phase diagram and then provide a qualitative characterization of them.

To that end, consider the set of points for which $\dot{x}=0$, a curve known as the $\dot{x}=0$ isocline. The isocline is easy to derive given the simple functional form of the state equation in Eq. (26), and is given by $q=R$. Hence the $\dot{x}=0$ isocline is a horizontal line at the constant value of $R>0$ in the $(x, q)$ phase space. Clearly, if $q>R$ then $\dot{x}<0$, while if $q<R$ then $\dot{x}>0$. This motion has been indicated by placing a (+) below the $\dot{x}=0$ isocline and a (-) above it. The signs show how the stock of water changes over time as a function of the extraction rate of water.

From Eq. (27) and the fact that $C_{q q}(x, q)>0$, it follows that the $\dot{q}=0$ isocline is defined implicitly by $A(x, q ; \boldsymbol{\theta}) \stackrel{\text { def }}{=} C_{q x}(x, q)[R-q]+r\left[p-C_{q}(x, q)\right]+C_{x}(x, q)=0$. Furthermore, because $A_{q}\left(x^{s}(\boldsymbol{\theta}), q^{s}(\boldsymbol{\theta}) ; \boldsymbol{\theta}\right)=-r C_{q q}\left(x^{s}(\boldsymbol{\theta}), q^{s}(\boldsymbol{\theta})\right)<0$, the implicit function theorem implies that the implicit equation $A(x, q ; \boldsymbol{\theta})=0$ can in principle be solved for $q$ in a neighborhood of the steady state solution $\left(x^{s}(\boldsymbol{\theta}), q^{s}(\boldsymbol{\theta})\right)$ to get $q=Q(x ; \boldsymbol{\theta})$, thereby yielding the equation for the $\dot{q}=0$ isocline. By the implicit function theorem, the slope of the $\dot{q}=0$ isocline in a neighborhood of the steady state is

$$
\left.\frac{\partial Q}{\partial x}\right|_{\substack{x=0 \\ \dot{q}=0}}=\frac{-A_{x}\left(x^{s}(\boldsymbol{\theta}), q^{s}(\boldsymbol{\theta}) ; \boldsymbol{\theta}\right)}{A_{q}\left(x^{s}(\boldsymbol{\theta}), q^{s}(\boldsymbol{\theta}) ; \boldsymbol{\theta}\right)}=\frac{r C_{q x}\left(x^{s}(\boldsymbol{\theta}), q^{s}(\boldsymbol{\theta})\right)-C_{x x}\left(x^{s}(\boldsymbol{\theta}), q^{s}(\boldsymbol{\theta})\right)}{-r C_{q q}\left(x^{s}(\boldsymbol{\theta}), q^{s}(\boldsymbol{\theta})\right)}>0 .
$$


Thus the $\dot{q}=0$ isocline is positively sloped in a neighborhood of the steady state. Because $\partial \dot{q} /\left.\partial q\right|_{\substack{\dot{x}=0 \\ \dot{q}=0}}>0$ from Eq. (28), $q$ is increasing over time for points above the $\dot{q}=0$ isocline in a neighborhood of the steady state, hence the (+) above the $\dot{q}=0$ isocline, and similarly for points below the isocline. Putting the information deduced in this and the preceding paragraph together, the phase diagram corresponding to Eqs. (26) and (27) may be derived. The result is exhibited in Figure 1.

The stable manifold in Figure 1 represents the solution to problem (17). If $x_{0}<x^{s}(\boldsymbol{\theta})$, then the unique solution is qualitatively described by the trajectory that approaches the steady state from the southwest. In this case, the stock of water and the extraction rate of water increase monotonically over time. Given the relatively small initial stock of water, the optimal plan is to keep the extraction rate below the natural recharge rate, thereby permitting the stock of water to build up over time until it "reaches" the steady state. If, however, $x_{0}>x^{s}(\boldsymbol{\theta})$, then the unique solution is qualitatively described by the trajectory that approaches the steady state from the northeast. In this situation the stock of water and the extraction rate decline monotonically over time towards the steady state because of the relatively large initial stock of water. It should be noted, however, that as long as $x_{0} \neq x^{s}(\boldsymbol{\theta})$, the optimal trajectory never reaches the steady state in finite time, i.e., the optimal trajectory only asymptotically approaches the steady state.

The preceding qualitative characterization of the solution is but the first step in a complete qualitative analysis of problem (17). This may come as a surprise given that it is impossible to derive an explicit solution of problem (17). In what follows, three complementary qualitative results will be derived, to wit, a steady state comparative statics result, and its corresponding local comparative dynamics and dynamic envelope results. The parameter of interest is the price of the extracted water $p>0$.

The comparative statics of the steady state solution $\left(x^{s}(\boldsymbol{\theta}), q^{s}(\boldsymbol{\theta})\right)$ must be derived first, as one must know how the terminal position of the optimal path changes before one can determine how the optimal path to the destination changes. Recall that the steady state solution 
$\left(x^{s}(\boldsymbol{\theta}), q^{s}(\boldsymbol{\theta})\right)$ is the simultaneous solution of Eqs. (26) and (27) when $\dot{x}=0$ and $\dot{q}=0$, i.e., it is the solution of

$$
\begin{gathered}
R-q=0, \\
r\left[p-C_{q}(x, q)\right]+C_{x}(x, q)=0,
\end{gathered}
$$

as $C_{q q}(x, q)>0$. A general procedure to derive the steady state comparative statics is to use the implicit function theorem, that is, substitute $\left(x^{s}(\boldsymbol{\theta}), q^{s}(\boldsymbol{\theta})\right)$ in Eqs. (30) and (31) to create identities in $\boldsymbol{\theta}$, differentiate the identities with respect to the parameter of interest, and then solve the resulting linear system using the information contained in $J_{d}$ to help sign the comparative statics expressions. For problem (17), this general procedure may be simplified due to the structure of the state equation.

From Eq. (30), it is clear that an explicit solution for the steady state extraction rate may be found, namely, $q^{s}(\boldsymbol{\theta})=R$. As a result, $\partial q^{s}(\boldsymbol{\theta}) / \partial p \equiv 0$, that is, the steady state extraction rate is unaffected by an increase in the price of the extracted water. Thus there is no classical supply response in the steady state. This is a result of the simple structure of the state equation.

Given the above conclusion, it follows that the steady state stock of water $x^{s}(\boldsymbol{\theta})$ is defined implicitly by $r\left[p-C_{q}(x, R)\right]+C_{x}(x, R)=0$. As a result, upon differentiating the identity $r\left[p-C_{q}\left(x^{s}(\boldsymbol{\theta}), R\right)\right]+C_{x}\left(x^{s}(\boldsymbol{\theta}), R\right) \equiv 0$ with respect to $p$, it follows that

$$
\frac{\partial x^{s}(\boldsymbol{\theta})}{\partial p}=\frac{-r}{-r C_{q x}\left(x^{s}(\boldsymbol{\theta}), R\right)+C_{x x}\left(x^{s}(\boldsymbol{\theta}), R\right)}<0 .
$$

Thus an increase in the price of extracted water decreases the steady state stock of water. This appears to be a surprising result, as it was deduced in the preceding paragraph that an increase in price has no effect on the steady state extraction rate. In other words, how can the steady state stock of water be lower when the price increases if the steady state extraction rate is unchanged? A local comparative dynamics analysis clarifies what is going on.

To begin, notice that the $\dot{x}=0$ isocline, viz., $q=R$, is independent of $p$, and is therefore unaffected by an increase in $p$. Given this fact and $\partial x^{s}(\boldsymbol{\theta}) / \partial p<0$, it follows that the $\dot{q}=0$ isocline shifts left when $p$ increases. The leftward shift in the $\dot{q}=0$ isocline may also be de- 
duced by applying the implicit function theorem to $A(x, q ; \boldsymbol{\theta})=0$, the implicit equation defining the $\dot{q}=0$ isocline.

Putting the information in the preceding paragraph together permits the construction of the local comparative dynamics phase diagram displayed in Figure 2. In constructing the diagram, observe that (i) the vector field around each steady state is qualitatively identical and may be inferred from Figure 1, (ii) the initial value of the water stock is taken to be its steady state value before the price increase, scilicet, $x_{\text {old }}^{s}$, and (iii) the optimal solution converges to a new steady state defined by the higher price, namely, $\left(x_{\text {new }}^{s}, R\right)$. Given these features, the local comparative dynamics of a price increase may be read off Figure 2.

Because the state variable is fixed at any point in time, the instant that the price increases, only the extraction rate changes. The initial change in the extraction rate must place it on the new stable manifold that approaches the new steady state from the northeast, as this is the only trajectory that can be reached from the old steady state that converges to the new steady state. Hence the initial effect of the price increase yields a positive supply response. Seeing as the extraction rate exceeds the natural recharge rate all along the new stable manifold, and only asymptotically returns to its former steady state value, the stock of water is drawn down over time. Thus the seemingly unusual steady state comparative statics results—no change in the extraction rate but a decline in the stock of water-are rendered intuitive once the local comparative dynamics are derived.

The last matter is to determine the effect the price increase has on the welfare of the firm. By using a dynamic analogue of the prototype envelope theorem, this issue may be investigated. In the present case, the dynamic envelope theorem yields the following recipe and result:

$$
\frac{\partial \Pi(\boldsymbol{\beta})}{\partial p}=\left.\int_{0}^{+\infty} e^{-r t} \frac{\partial H}{\partial p}(x(t), q(t), \lambda(t) ; p, R)\right|_{\substack{\text { optimal } \\ \text { solution }}} d t=\int_{0}^{+\infty} e^{-r t} q^{*}(t ; \boldsymbol{\beta}) d t>0 .
$$

Not surprisingly, the maximum present discounted value of the firm's profit increases as a result of the increase in the price of the extracted water. Note that the dynamic envelope result recovers the cumulative discounted value of the optimal extraction rate. 


\section{SUMMARY AND CONCLUSION}

Optimal control theory is one of the most important mathematical tools used by natural resource economists to analyze continuous-time dynamic optimization problems. Having said that, it is important to understand that even though the theorems stated in this chapter are applicable to many of the typical optimal control problems encountered in natural resource economics, they are not the most general available. Indeed, there are several complications that can and do occur when formulating such problems. For example, some natural resource economics problems are linear in the control variables, while others have equality or inequality constraints on the control and state variables, or include equality or inequality constraints on just the state variables, the last being the most difficult complication to address. In each of these cases, more general theorems are required in order to find and characterize the solution.

It is also worthwhile to remember that the two examples were not chosen because of their generality or realism, but to demonstrate how to (i) solve for the explicit solution of an optimal control problem and conduct a qualitative analysis, and (ii) derive a qualitative characterization of the solution of an optimal control problem when the functional forms of the integrand and transitions functions are not given.

\section{Further Reading}

Barrett, S. (1991), "Optimal Soil Conservation and the Reform of Agricultural Pricing Policies," Journal of Development Economics 36, 167-187.

Caputo, M.R. (1989), "The Qualitative Content of Renewable Resource Models," Natural Resource Modeling 3, 241-259.

Caputo, M.R. (1990), "How to do Comparative Dynamics on the Back of an Envelope in Optimal Control Theory," Journal of Economic Dynamics and Control 14, 655-683.

Caputo, M.R. (1990), "A Qualitative Characterization of the Competitive Nonrenewable Resource Extracting Firm," Journal of Environmental Economics and Management 18, 206-206. 
Caputo, M.R. (2003), “The Comparative Dynamics of Closed-Loop Controls for Discounted Infinite Horizon Optimal Control Problems," Journal of Economic Dynamics and Control 27, 1335-1365.

Caputo, M.R. (2005), Foundations of Dynamic Economic Analysis: Optimal Control Theory and Applications, Cambridge University Press, Cambridge.

Dockner, E. (1985), "Local Stability Analysis in Optimal Control Problems with Two State Variables," in Feichtinger, G (ed.), Optimal Control Theory and Economic Analysis, Vol. 2, North-Holland, Amsterdam.

Farzin, Y.H. (1992), "The Time Path of Scarcity Rent in the Theory of Exhaustible Resources," Economic Journal 102, 813-830.

Grass, D., Caulkins, J.P., Feichtinger, G., Tragler, G., and Behrens, D.A. (2008), “Optimal Control of Nonlinear Processes: With Applications in Drugs, Corruption, and Terror," Springer, Heidelberg.

Krautkraemer, J.A. (1998), "Nonrenewable Resource Scarcity," Journal of Economic Literature 36, 2065-2107.

LaFrance, J.T. and Barney, L.D. (1990), “The Envelope Theorem in Dynamic Optimization,” Journal of Economic Dynamics and Control 15, 355-385.

Léonard, D. (1987), “Costate Variables Correctly Value Stocks at Each Instant: A Proof,” Journal of Economic Dynamics and Control 11, 117-122.

Léonard, D. and Van Long, N. (1992), Optimal Control Theory and Static Optimization in Economics, Cambridge University Press, New York.

Oniki, H. (1973), "Comparative Dynamics (Sensitivity Analysis) in Optimal Control Theory," Journal of Economic Theory 6, 265-283.

Seierstad, A. and K. Sydsæter (1987), Optimal Control Theory with Economic Applications, Elsevier Science Publishers, New York.

Tahvonen, O. (1991), "On the Dynamics of Renewable Resource Harvesting and Pollution Control," Environmental and Resource Economics 1, 97-117. 


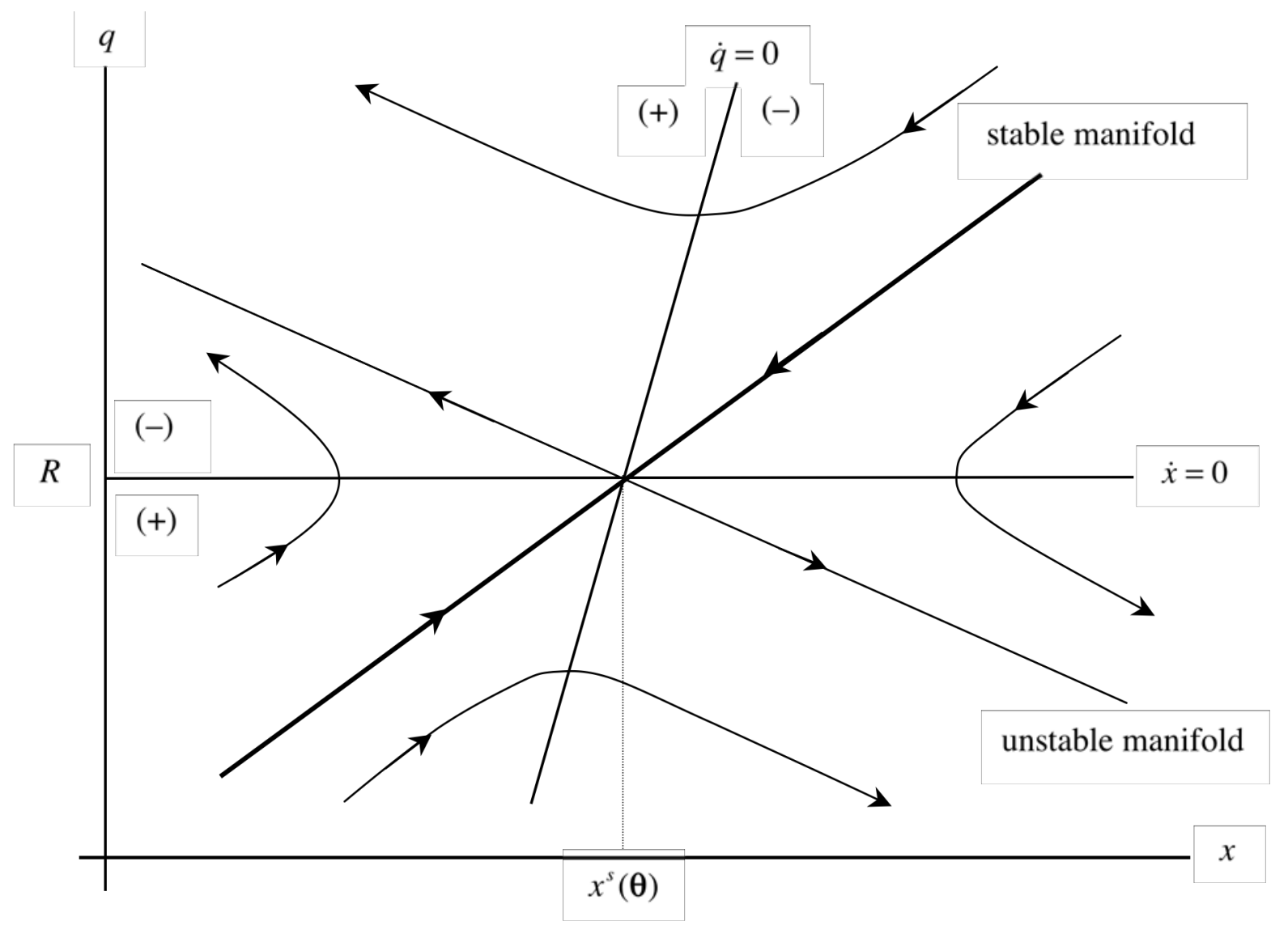

Figure 1 


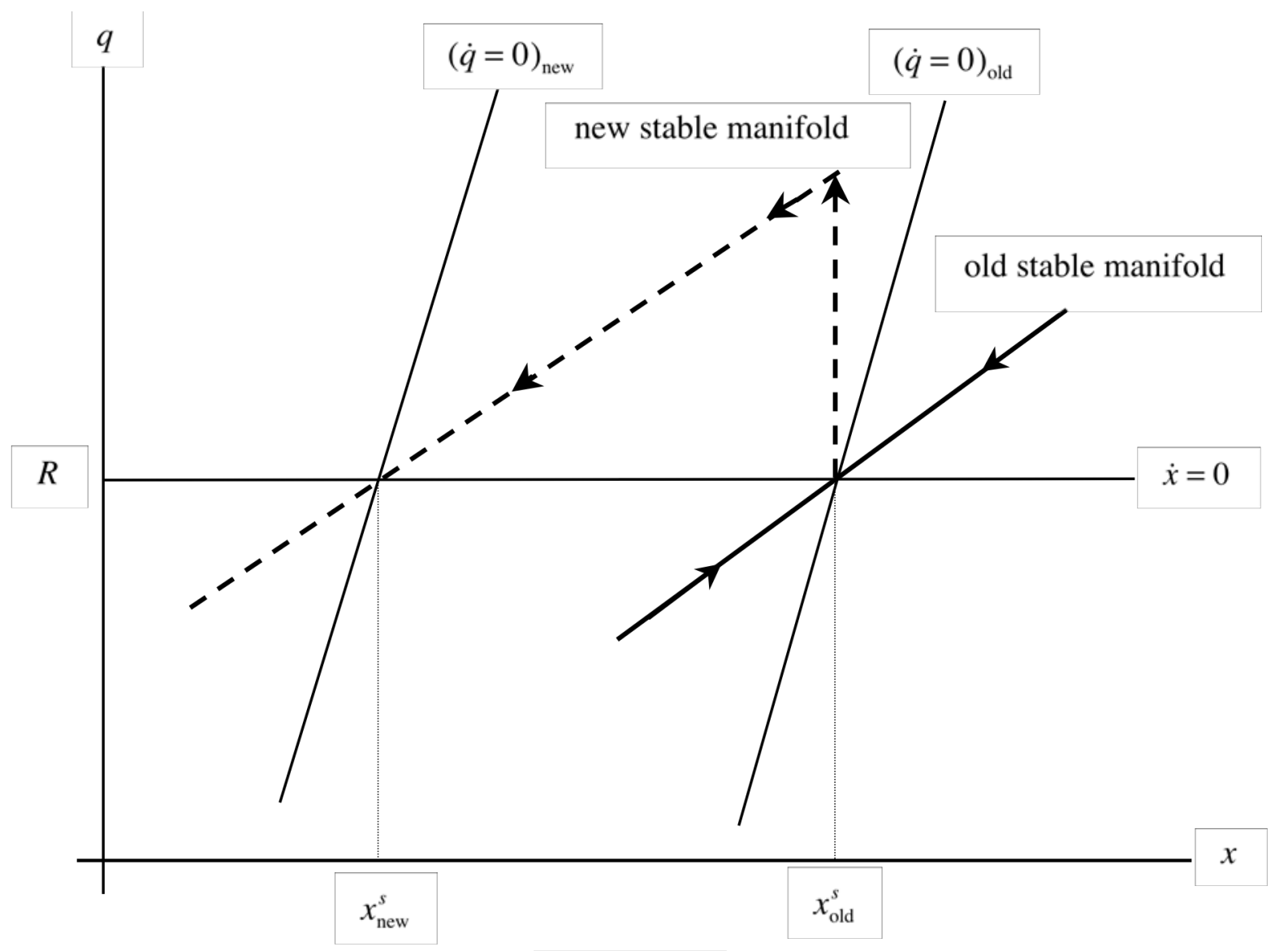

Figure 2 